\title{
Method for studying the role of indigenous cervical flora in colonisation by Neisseria gonorrhoeae
}

\author{
M. E. MCBRIDE, W. C. DUNCAN, AND J. M. KNOX \\ From the Departments of Dermatology and Microbiology, Baylor College of Medicine, \\ Texas Medical Center, Houston, Texas, USA
}

SUMMARY A method for quantitating cervical flora has been evaluated statistically and used to study the bacterial flora of the cervix in 14 women sexually exposed to men with gonococcal urethritis. A comparison was made between those women who subsequently became colonised with Neisseria gonorrhoeae and those who did not to determine whether either total microbial populations or the different species present could be related to colonisation by $N$. gonorrhoeae. Two control groups of healthy women, one of patients from a public clinic and the other of patients from a private practice, were studied in the same way. Normal flora isolates were tested in vitro for antagonism or synergism toward $N$. gonorrhoeae or both. Cervical flora was characterised in all patient groups by wide variations between individuals, both in type and numbers of organisms. No significant differences were found in total bacterial populations or in the number of species isolated from the cervix between patient groups. Populations of $N$. gonorrhoeae ranged from less than 10 bacteria to $\log _{10} 4 \cdot 36$. Only one normal flora isolate, a strain of Streptococcus viridans isolated from a woman exposed to but not infected by $N$. gonorrhoeae, demonstrated inhibition of growth towards $N$. gonorrhoeae.

\section{Introduction}

There has been increasing interest in the importance of the microbial flora of normal healthy individuals in maintaining a stable ecological balance which might protect against colonisation by pathogenic micro-organisms. This type of control can occur in different ways: as antagonism between microbial species (or bacterial interference); or by the availability of the ecological space to invasion, which occurs in the absence or suppression of normal flora. Bacterial interference has been described in a variety of human ecosystems, and includes the organisms associated with dental plaque (Homberg and Halkinder, 1972), skin microflora (Selwyn, 1975), and the flora of the respiratory tract (Sprunt et al, 1971; Crowe et al, 1973), while suppression of normal flora as a result of antibiotic treatment has been known to result in colonisation by opportunists.

The complexity of the microflora of the female genital tract makes it difficult to assess its protective

Address for reprints: Dr M. E. McBride, Department of Dermatology, Baylor College of Medicine, Texas Medical Center, Houston, Texas 77030, USA

Received for publication 16 March 1978 role against infection. The composition of vaginal and cervical flora has been a subject of interest since Döderlein's classical description of Lactobacillus in 1892. Hite et al (1947) reviewed some of the early literature while Galask et al (1976) reevaluated the role of vaginal flora in disease. A comprehensive overview of the problems of studying the microflora of the female genital tract as an ecosystem was presented by Hurley et al (1974), and more recent definitive studies using quantitative techniques (Bartlett et al, 1977; Levison et al, 1977) have emphasised the complexity of the flora of the female genital tract.

Although bacterial interference has been described between Neisseria gonorrhoeae and Staphylococcus epidermidis (Kraus and Ellison, 1974), Escherichia coli (Kraus et al, 1976), and Candida albicans (Hipp et al, 1974) studies indicating shifts in population groups related to infection have been few. deLouvois et al (1975) were able to correlate statistically the increase in isolation of Trichomonas vaginalis, Mycoplasma hominis, and Bacteroides species with a decrease of Lactobacillus. The observation that decreased frequency of isolation of Lactobacillus correlated with vaginitis and 
isolation of $T$. vaginalis was also recorded by Gordon et al (1966).

The purpose of this study has been to attempt to identify individual differences in the microflora of the cervix in women exposed to, but not necessarily colonised by, $N$. gonorrhoeae with the aim of identifying organisms which might exert a protective effect. To do this a method for quantitation of the cervical flora has been devised and evaluated statistically. Two other groups of patients have been included in the study to provide a baseline for normal variability: healthy women attending a public premarital clinic and healthy women from a private gynaecological practice. Bacterial isolates from each of these groups have been examined for interference with $N$. gonorrhoeae in vitro.

\section{Materials and methods}

\section{QUANTITATION OF CERVICAL FLORA}

Study population

A total of 45 patients were studied. Of these, 14 women attending the City of Houston Public Health Clinic were known recent sexual contacts of men with acute gonococcal urethritis; 11 were presumably healthy women attending the premarital clinic; and 20 were presumably healthy patients from a private obstetrical practice reporting for a routine cervical cytological smear. None of the patients had taken antibiotics in the preceding three weeks, and none had douched within 24 hours.

\section{Sampling methods}

Two Dacron-tipped swabs were used to obtain duplicate samples from the cervical os using a speculum. The swabs were immediately placed in Stuart's medium and transported to the laboratory within a maximum of three hours from the time the cultures were taken. A third cervical culture was plated on Thayer-Martin medium for routine examination by the Houston Public Health Laboratory for $N$. gonorrhoeae. Identical sampling methods were used for each group of women.

\section{Culture methods}

Each duplicate swab was placed in $1.0 \mathrm{ml}$ of phosphate-buffered saline $(\mathrm{pH} 7 \cdot 2)$ and shaken on a Vortex junior mixer for one minute. Smears of this suspension were made for Gram staining. A $0 \cdot 1 \mathrm{ml}$ aliquot was inoculated directly on to a Casman's sheep blood agar plate (Casman's Medium Base, Difco) and Rogosa's medium (Rogosa's SL Agar, Difco) and streaked. The $1.0 \mathrm{ml}$ suspension was further diluted using tenfold dilutions to $10^{4}$. Each dilution was inoculated on to the surface of a
Casman's sheep blood agar plate using a calibrated dropping pipette and six drops per plate. Differential media were used randomly to determine their value; these included chocolate agar, Thayer-Martin medium, tomato juice agar (Difco), and Rogosa's agar, which were found to be the most useful. Casman's sheep blood agar was superior to chocolate agar for the isolation of fastidious organisms, and Rogosa's agar was found to the most useful for growth of Lactobacillus. Other selective media did not provide additional information. In certain patients duplicate plates of Casman's sheep blood agar were inoculated for growth in an anaerobic jar (Gaspak). Rogosa's agar was incubated anaerobically using the Gaspak system at $37^{\circ} \mathrm{C}$. Aerobic incubation supported only a very light growth of organisms such as E. coli, Micrococcus, et cetera. Growth was visible at $\mathbf{4 8}$ hours, but further colonial differentiation could be observed at 72 hours, particularly the development of haemolysis. The appropriate culture dilution (usually $10^{3}$ or $10^{4}$ ) was chosen with the aid of a stereoscopic microscope; it was then possible to do a differential count based on colonial morphology. Species present in low numbers were counted from the plate streaked with $0.1 \mathrm{ml}$ inoculum. Identification and characterisation were carried out using methods of Cowan and Steele (1975).

At the Public Health Laboratory suspected colonies from cervical cultures were Gram-stained, and the oxidase reaction was determined. Where this reaction was positive and typical Gramnegative diplococci were present the organism was considered to be $N$. gonorrhoeae.

\section{Statistical methods}

A paired $t$ statistic was used to compare total counts in duplicate specimens. Multiple regression evaluated by an $\mathrm{F}$ statistic was used to determine the correlation between the populations of species isolated from the duplicate samples after correcting for the total counts of the duplicates.

\section{BACTERIAL INTERFERENCE Screening method 1}

A suspension was made of a fresh clinical isolate of $N$. gonorrhoeae in phosphate-buffered saline $(\mathrm{pH}$ 7.2) and diluted from $10^{1}$ to $10^{4}$. An $0 \cdot 1 \mathrm{ml}$ aliquot of each dilution was plated on the surface of Casman's sheep blood agar plates. Isolates of normal flora were spotted on the surface of the $N$. gonorrhoeae lawns. The plates were incubated in a $\mathrm{CO}_{2}$ jar at $37^{\circ} \mathrm{C}$ and examined at 24 and 48 hours. 


\section{Screening method 2}

Lawns were prepared on Casman's sheep blood agar with isolates of normal flora organisms. Dilutions of an overnight culture of a fresh isolate of $N$. gonorrhoeae were spotted on to the lawns by means of a calibrated pipette delivering a constant inoculum. Control plates of Casman's sheep blood without normal flora were inoculated in the same way. Plates were incubated in a $\mathrm{CO}_{2}$ jar at $37^{\circ} \mathrm{C}$ and examined at 24 and 48 hours for inhibition or synergism of growth of $N$. gonorrhoeae or both.

\section{Growth curves of mixed populations}

Normal flora organisms giving some indication of synergism or antagonism were chosen for growth in mixed cultures. Preliminary experiments were done to achieve an equivalent concentration of each species of bacteria as inoculum. Screw-topped test tubes containing $50 \mathrm{ml}$ of Casman's broth containing $5 \%$ sheep's blood were inoculated at $37^{\circ} \mathrm{C}$ for five days. Control pure cultures of each of the species were inoculated and incubated under the same conditions. Aliquots were removed from these cultures daily, diluted in phosphate-buffered saline and inoculated on to Casman's sheep blood agar and Thayer-Martin medium by means of a calibrated dropping pipette. These plates were incubated in a $\mathrm{CO}_{2}$ jar at $37^{\circ} \mathrm{C}$ for 48 hours, at which time a colony count was done and a growth curve plotted.

\section{Results}

Since the cervical specimen was suspended in diluent before plating, organisms present in low numbers were not cultured. When $0.1 \mathrm{ml}$ of inoculum suspension was used on a streak plate common species associated with genital tract flora-for example, $E$. coli and other enteric organisms, Staph, epidermidis, Micrococcus species, and diptheroids-could be readily enumerated. These organisms represent a relatively low proportion of the total flora and seem to be more of a 'contaminant' flora since further dilutions of the sample yielded high populations of more fastidious organisms. Figures 1 and 2 show growth from inoculum delivered from a calibrated dropping pipette of $10^{2}(\times 1.3$ magnification $)$ and $10^{4}(\times 2.5$

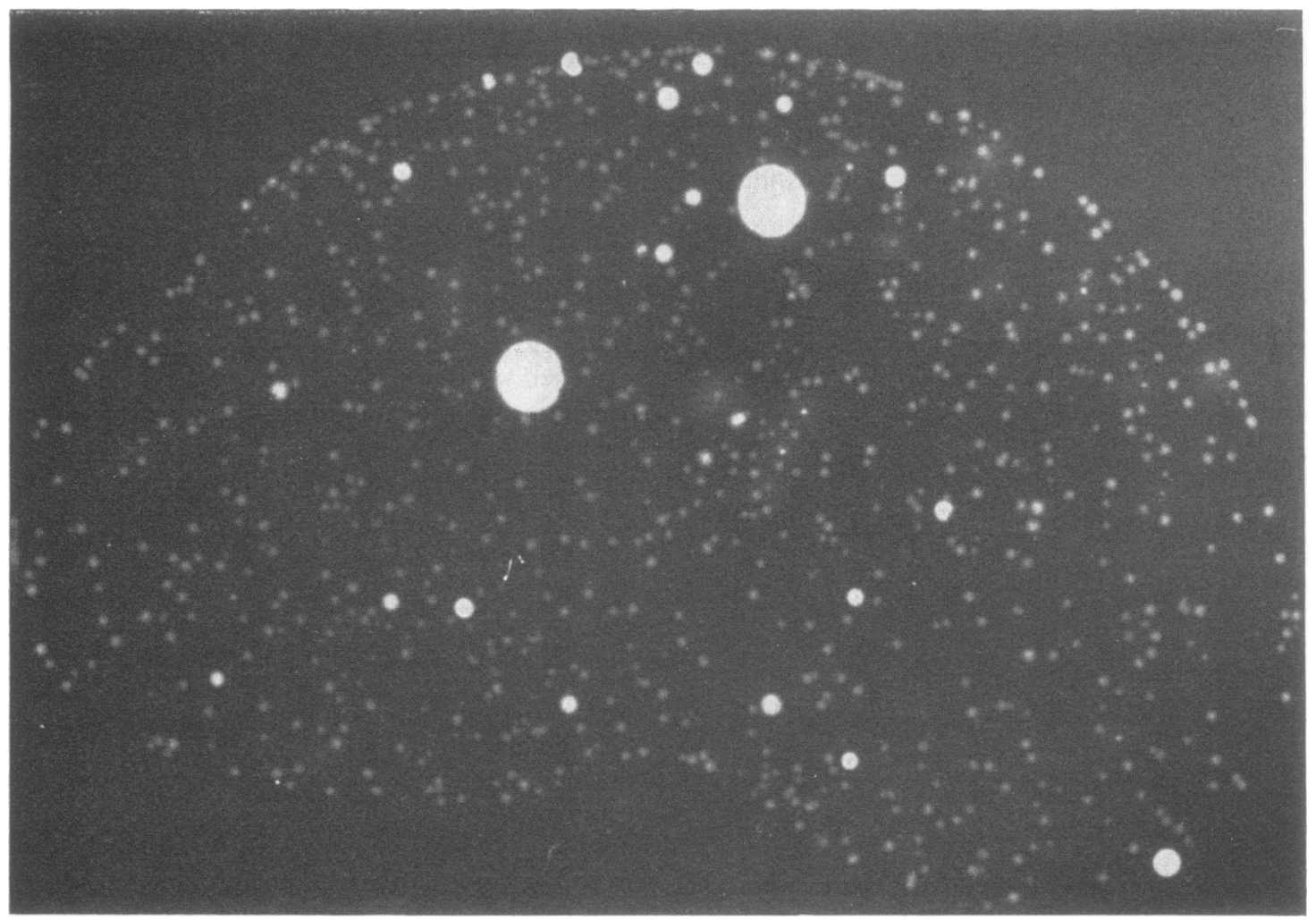

Fig. 1 Casman's sheep blood agar plate inoculated with $10^{2}$ dilution of cervical specimen $(\times 1 \cdot 3$ magnification) 
magnification) dilution of a typical specimen. Different colony types are evident, and a differential count can be done on the basis of colonial morphology. The flora was predominantly $\mathrm{CO}_{2}$ dependent and facultatively anaerobic, with the exception of Bacteroides, some Lactobacillus species, Veillonella species, and a group of Gram-negative cocci which were strictly anaerobic. Identifiable genera found in high populations included a variety of streptococci, Eikenella, Haemophilus vaginalis, and other Haemophilus species. Many isolates did not readily conform to identification schemes.

\section{Evaluation of quantitative method}

For statistical evaluation of the methods the results from all patient groups were pooled. Table 1 shows the range of microbial populations in duplicate samples A and B taken from the cervix. The cervical flora was found to be very variable between individuals both in total populations and in different species present, but duplicate cultures of the cervix taken in a uniform manner were found to contain statistically comparable microbial populations. The distribution of the total populations from duplicate cultures is shown in Figure 3. There is a normal distribution of populations, the majority of patients having populations ranging between $\log _{10} 4$ to $\log _{10} 7$ colony-forming units (cfu) per $\mathrm{ml}$ per sample inclusive.

Comparison of populations of different species isolated from duplicate cervical cultures

A total of 115 isolates was obtained from the 45 patients, and only three of these did not appear in the duplicate cultures. There were 12 patients with

Table 1 Comparison of microbial populations of duplicate cultures of the cervix

\begin{tabular}{lllll}
\hline \multirow{5}{*}{$\begin{array}{l}\text { Duplicate } \\
\text { cultures }\end{array}$} & \multicolumn{4}{l}{$\begin{array}{l}\text { Microbial populations } \\
\left(\log _{10} c f u / m l\right.\end{array}$} \\
\cline { 2 - 5 } & Range & Mean & $S D$ & $S E$ \\
\hline Sample A & $2 \cdot 54-7 \cdot 55$ & $5 \cdot 36$ & $1 \cdot 15$ & $0 \cdot 18$ \\
Sample B & $2 \cdot 65-7 \cdot 14$ & $5 \cdot 40$ & $1 \cdot 12$ & $0 \cdot 19$ \\
\hline
\end{tabular}

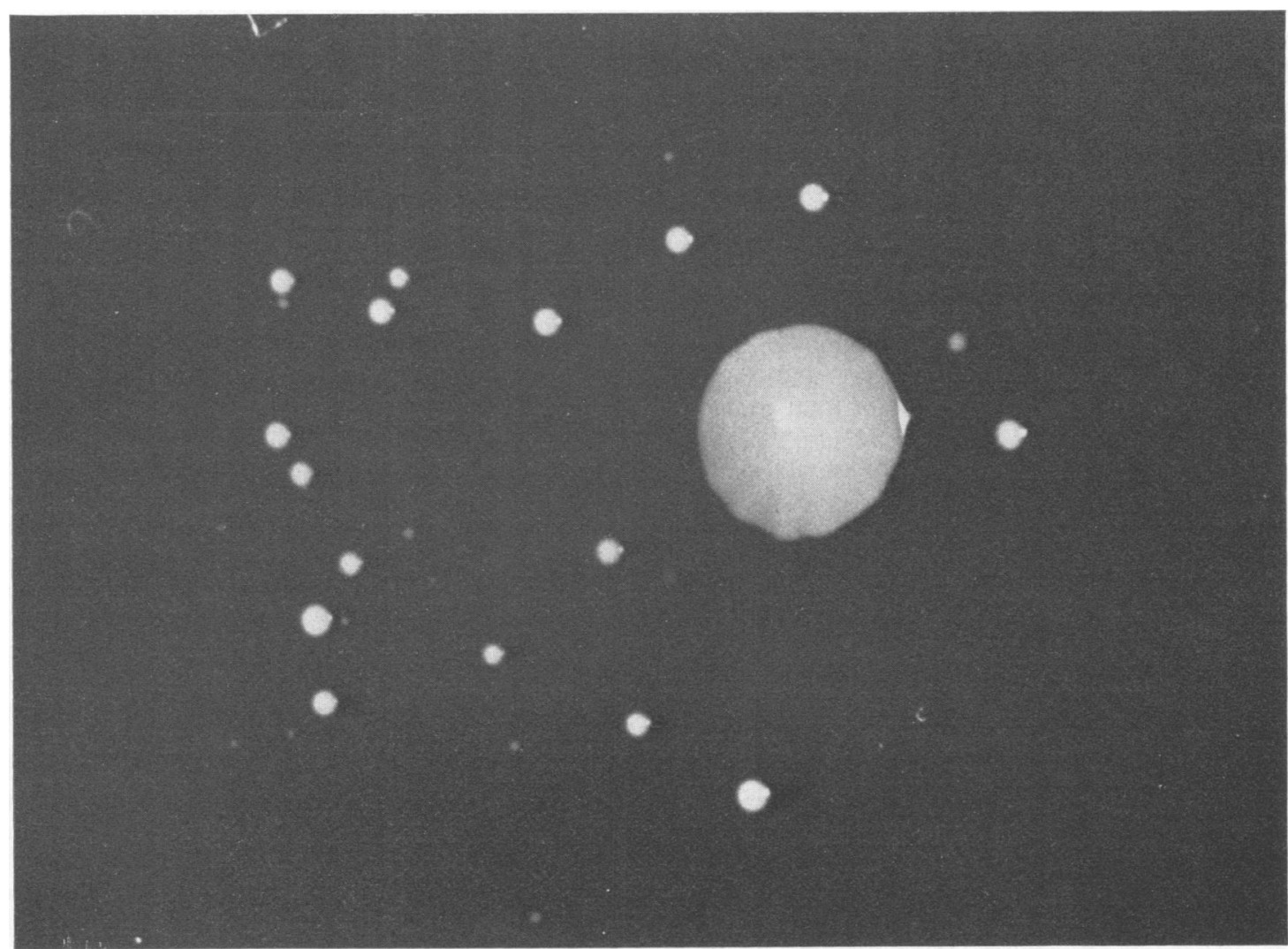

Fig. 2 Casman's sheep blood agar plate inoculated with $10^{4}$ dilution of cervical specimen $(\times 2 \cdot 2$ magnification) 


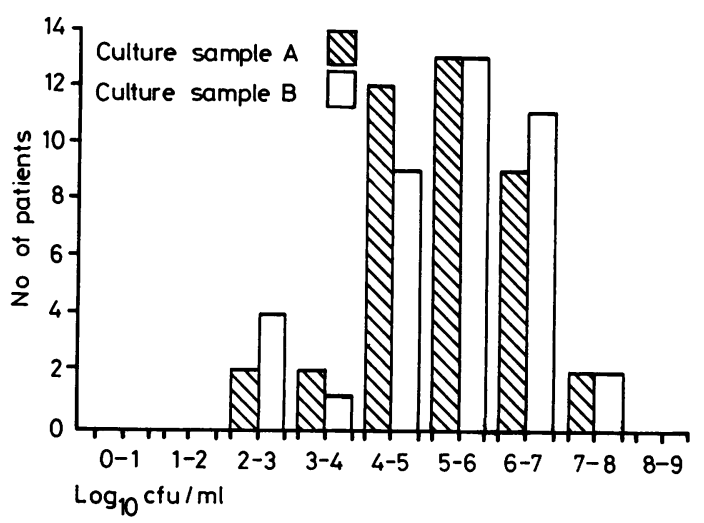

Fig. 3 Distribution of total populations from duplicate cultures from the cervical os

pure cultures of a single organism, and the largest number of isolates per culture was seven. The populations of the individual species in duplicate cultures were compared by means of the method of multiple regression. After regressing the individual species of culture sample $B$ from the total populations of sample $A$ and duplicate sample $B$, there remained a large regression coefficient $(R)$ for the individual species (B). R square for the total for sample A plus the total for sample $B$ was $\mathbf{0 . 3 2}$, while for total populations for sample A plus total populations for sample $B$ plus the values for the particular species in sample A was 0.86 . The F statistic for an individual species was 397.9 on 1 with 106 degrees of freedom, which is significant at the 0.001 level. Thus the populations of species isolated from duplicate cultures $\mathbf{A}$ and $\mathbf{B}$ were highly correlated even correcting for total counts.

\section{Comparison of bacterial populations in different patient groups}

The total bacterial populations were calculated for each patient group to determine whether individuals with lower populations of indigenous microfiora were more susceptible to colonisation by $N$. gonorrhoeae. Similarly, the importance of a more varied flora, as demonstrated by the number of different species isolated, was examined. Bacterial populations of the cervix of the three patient groups studied (healthy practice patients; healthy clinic patients; and asymptomatic sexual contacts of $N$. gonorrhoeae) are shown in Table 2 . The range and mean values of the total population in the specimen were comparable between the three groups. The results from the 14 contacts of $N$. gonorrhoeae were broken down further into those with positive results from cultures for $N$. gonorrhoeae and those with negative results. The presence or absence of $N$. gonorrhoeae in the cultures was determined independently at the Public Health Laboratory and correlated with the results of this study; half the patients were found to harbour $N$. gonorrhoeae. The results are shown in Table 3. Although the microbial populations per sample

Table 2 Comparison of bacterial populations of the cervix in three patient groups

\begin{tabular}{|c|c|c|c|c|c|}
\hline \multirow{2}{*}{$\begin{array}{l}\text { Patient } \\
\text { groups }\end{array}$} & \multirow{2}{*}{$\begin{array}{l}\text { No. of } \\
\text { patients }\end{array}$} & \multicolumn{2}{|c|}{$\begin{array}{l}\text { Microbial populations } \\
\text { (log10cfu/specimen) }\end{array}$} & \multicolumn{2}{|c|}{ No. of isolates } \\
\hline & & Range & Mean & Range & Mean \\
\hline \multirow{3}{*}{$\begin{array}{l}\text { Contacts of } \\
N . \text { gonorrhoeae } \\
\text { Healthy clinic } \\
\text { patients } \\
\text { Healthy private } \\
\text { patients }\end{array}$} & 14 & $2 \cdot 54-7 \cdot 14$ & $5 \cdot 39$ & $1-4$ & $2 \cdot 78$ \\
\hline & 11 & $2 \cdot 91-6.64$ & $5 \cdot 09$ & $1-6$ & 3.09 \\
\hline & 20 & $1 \cdot 69-7 \cdot 55$ & $5 \cdot 12$ & $1-7$ & $3 \cdot 35$ \\
\hline
\end{tabular}

Table 3 Comparison of bacterial populations of the cervix in 14 sexual contacts of $\mathrm{N}$. gonorrhoeae

\begin{tabular}{|c|c|c|c|c|c|c|}
\hline \multirow[b]{2}{*}{ Patient group } & \multirow{2}{*}{$\begin{array}{l}\text { No. of } \\
\text { patients }\end{array}$} & \multicolumn{3}{|c|}{$\begin{array}{l}\text { Microbial populations } \\
\text { (log } 10 c f u / \text { specimen) }\end{array}$} & \multicolumn{2}{|c|}{ No. of isolates } \\
\hline & & Range & Mean & $S D$ & Range & Mean \\
\hline $\begin{array}{l}N \text {. gonorrhoeae } \\
\text { contact } \\
\text { culture-positive } \\
\text { culture-negative }\end{array}$ & & $\begin{array}{l}2 \cdot 54-6 \cdot 47 \\
4 \cdot 57-7 \cdot 14\end{array}$ & $\begin{array}{l}4.99 \\
5.94\end{array}$ & $\begin{array}{l}1 \cdot 14 \\
1 \cdot 40\end{array}$ & $\begin{array}{l}1-4 \\
1-4\end{array}$ & $\begin{array}{l}2 \cdot 85 \\
2 \cdot 71\end{array}$ \\
\hline
\end{tabular}

were lower in patients colonised by $N$. gonorrhoeae, with a mean of $4.99 \log _{10}$ cfu per sample compared with $5.94 \log _{10}$ cfu per sample from those patients who were not colonised, the difference was not statistically significant $(t=1 \cdot 276)$. In addition there was no difference in the number of isolates obtained from each sample between the two groups. A lower population of indigenous flora, therefore, did not appear to be a predisposing factor to colonisation by $N$. gonorrhoeae.

The relative populations of $N$. gonorrhoeae to the total bacterial population of the specimen is shown in Table 4. Although three of the seven patients had relatively high populations of $N$. gonorrhoeae in their cervical cultures, none had pure cultures. Four patients had a population of $N$. gonorrhoeae of less than 10 bacteria. The high or low population of $N$. gonorrhoeae did not seem to correlate either directly or indirectly with high or low total populations. Similarly, the number of other species present appeared to bear no relationship to colonisation by $N$. gonorrhoeae. 
Table 4 Populations of Neisseria gonorrhoeae in cervical cultures

\begin{tabular}{llll}
\hline \multicolumn{3}{c}{$\begin{array}{l}\text { Microbial populations }\left(\log _{10} \mathrm{c} f u \mid\right. \\
\text { specimen) }\end{array}$} & \\
\cline { 2 - 4 } Case & Total & N. gonorrhoeae & No. of other species \\
\hline 14 & 2.54 & $<1.0$ & 1 \\
7 & 4.73 & 4.34 & 2 \\
16 & 4.91 & $<1.0$ & 4 \\
13 & 5.17 & 3.25 & 2 \\
15 & 5.50 & 4.36 & 3 \\
19 & 5.65 & $<1.0$ & 4 \\
8 & 6.47 & $<1.0$ & 4 \\
\hline
\end{tabular}

In an attempt to identify qualitative differences in flora between patient groups it was obvious that the differences between individuals were so great within each group that it would be very difficult to assess their relative importance. Figure 4 shows the flora of two healthy patients from the private practice group demonstrating the differences in both total populations and numbers of species present. These qualitative and quantitative differences were noted in each patient population studied. Lactobacillus was the only genus which showed recognisable differences between the three patient groups studied. The populations and incidence of isolation of Lactobacillus species from individuals exposed to $N$. gonorrhoeae in normal clinic patients and those from a private practice are shown in Table 5. Only one patient exposed to $N$. gonorrhoeae (but not infected) and two of the healthy clinic patients were colonised with Lactobacillus compared with 11 of the 20 private patients. Populations of Lactobacillus overall ranged from $\log _{10} 3.9$ to $6 \cdot 8$ cfu per specimen, representing $17.9 \%$ of the total bacteria present in one specimen to $100 \%$ in others.

Table 5 Comparison of populations of Lactobacillus species in three patient groups

\begin{tabular}{lll}
\hline & \multicolumn{2}{l}{ Lactobacillus species } \\
\cline { 2 - 3 } Patient group & $\begin{array}{l}\text { Population } \\
\left(\log _{10 c f u / m l} \text { specimen }\right)\end{array}$ & $\begin{array}{c}\% \text { of total bacterial } \\
\text { population }\end{array}$ \\
\hline Contacts of & & \\
N. gonorrhoeae & & \\
Case 10 & 6.08 & 98.3 \\
Healthy clinic patients & 6.84 & 100 \\
Case 5 & 5.25 & 100 \\
Case 9 & & \\
Healthy private & & 39.2 \\
patients & 4.23 & 38.0 \\
Case 6 & 4.6 & 100 \\
Case 7 & 5.1 & 19.6 \\
Case 10 & 3.9 & 43.9 \\
Case 12 & 6.2 & 100 \\
Case 13 & 5.2 & 23.0 \\
Case 15 & 5.6 & 47.5 \\
Case 16 & 5.9 & 17.9 \\
Case 17 & 5.4 & 100 \\
Case 18 & 3.5 & 62.0 \\
Case 19 & 6.2 & \\
Case 20 & & \\
\hline
\end{tabular}

$\mathbf{C}$

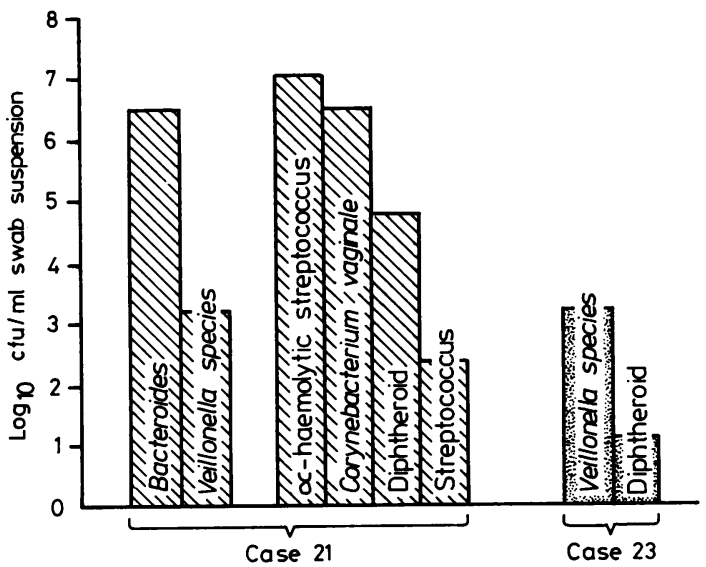

Fig. 4 Comparison of cervical flora in two healthy controls

\section{Bacterial interference}

The majority of the isolates obtained from each patient group were screened to demonstrate inhibitory or stimulatory activity towards $N$. gonorrhoeae in vitro. Only one isolate, a strain of Streptococcus viridans (obtained from a contact of $N$. gonorrhoeae), demonstrated inhibition towards $N$. gonorrhoeae. This patient had a negative culture result for $N$. gonorrhoeae. The growth of $N$. gonorrhoeae and the isolate of Str. viridans was followed in mixed liquid culture medium for four days, and the results are shown in Figure 5a. The growth of Str. viridans showed very little difference when grown in pure or mixed culture. The growth of $N$. gonorrhoeae in pure culture reached a high level and this was maintained for five days. In mixed culture, however, this organism did not achieve a high population level before showing a steady decline until no viable organisms were detected at four days.

The growth of $N$. gonorrhoeae in mixed culture with an organism isolated from normal flora from a patient colonised with $N$. gonorrhoeae is shown in Figure 5b. Growth of the normal flora isolate is not affected by $N$. gonorrhoeae. The growth of $N$. gonorrhoeae, while inhibited during the early stages of growth, within four days gradually reached populations only slightly lower than $N$. gonorrhoeae grown in pure culture. This isolate of normal flora is characteristic of cervical flora. It is a fastidious $\mathrm{CO}_{2}$-dependent $\beta$-haemolytic pinpoint colony showing Gram-variable-staining and pleomorphic cocco-bacillary types, oxidase- and catalase-negative and penicillin-sensitive.

Only one normal flora isolate, cultured from a private patient, showed inhibition by $N$. gonorrhoeae during the screening procedure. 


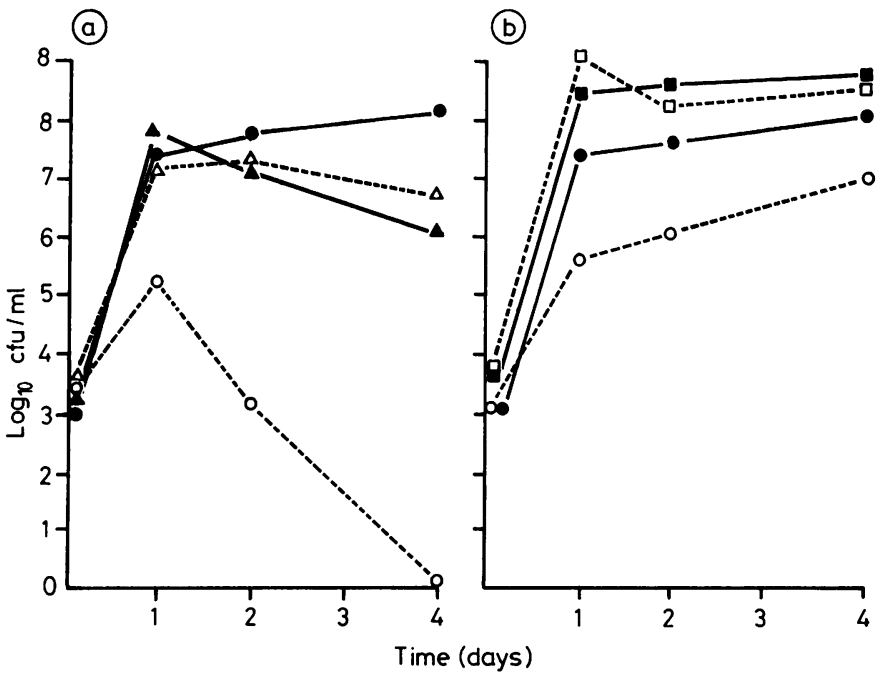

Fig. 5 Growth curves of mixed cultures of $\mathrm{N}$. gonorrhoeae and normal flora isolates

(a) $\longrightarrow N$. gonorrhoeae in pure culture; $0---0$ $N$. gonorrhoeae in mixed culture; $\Delta-\mathbf{\Delta}$ Streptococcus viridans in pure culture; $\triangle---\triangle$ Streptococcus viridans in mixed culture

\section{Discussion}

In an attempt to relate the role of normal flora of the cervix to colonisation by $N$. gonorrhoeae the reliability of a method of quantitation is essential. While the method described here can be considered only semi-quantitative, in as much as the sample size was not quantitated, the duplicate sampling procedure showed that it is possible-by uniform sampling - to obtain reliable quantitative results. Although the total microbial populations are semi-quantitative, the relative populations of different species are not in as much as they can be related to a fixed volume of sample material. When some of the differences in technique are taken into consideration the findings presented here concerning population levels of cervical flora are basically comparable to those described by Bartlett et al (1977).

The protective role of indigenous flora against colonisation by $N$. gonorrhoeae is not obvious either by the population levels utilising the ecological space or by the presence of any one particular species. Individuals with high populations of normal flora were colonised by $N$. gonorrhoeae while some exposed individuals with low cervical populations were not. In addition it was not possible to identify a characteristic flora from patients exposed to, but not infected by, $N$. gonorrhoeae. (b) $-N$. gonorrhoeae in pure culture; $0--0 N$ gonorrhoeae in mixed culture; $\square-\square$ normal flora isolate in pure culture; $\square---\square$ normal flora isolate in mixed culture

Although a number of different species of streptococcus were isolated, in only one patient was Str. viridans present and this showed inhibition towards growth of $N$. gonorrhoeae. This patient had contact with $N$. gonorrhoeae but was not infected. It would seem, therefore, that protection against infection by normal flora species would be a fortuitous event. Str. viridans has been known to demonstrate bacterial interference against group A Streptococcus pyogenes in the respiratory tract, where it occurs with a higher frequency of isolation and in high populations (Sprunt et al, 1971; Crowe et al, 1933). While the results of screening isolates for bacterial interference are largely negative, this may be a result of the in-vitro screening technique and may not reflect the in-vivo environment.

The individual variability, both quantitative and qualitative, in indigenous cervical flora emphasises the lack of information on the factors effecting normal flora. Thus, the importance of studying population fluctuations in individuals is obvious. Although a quantitative technique has served to elucidate the relative importance of various species comprising normal flora, our knowledge is still hampered by the lack of definition of many organisms present, many of which are extremely fastidious and do not conform to classification schemes.

The wide fluctuations in $N$. gonorrhoeae populations in women exposed to infection indicates 
some sort of innate protection, even though one of the most significant factors in the initiation of infection, the infectious dose, is unknown. The nature of this protection may be related to a multiplicity of factors, including the chemical environment and immunological factors. The protective role of the indigenous flora against $N$. gonorrhoeae cannot be dismissed by this limited study, but a method is presented for an area that should be pursued.

The technical assistance of Adelia McBride is gratefully acknowledged.

\section{References}

Bartlett, J. G., Onderdonk, A. B., Drude, E., Goldstein, C., Anderaka, M., Alpert, S., and McCormack, W. M. (1977). Quantitative bacteriology of the vaginal flora. Journal of Infectious Diseases, 136, 271-277.

Cowan, S. T. (1974). Cowan and Steel's Manual for the Identification of Medical Bacteria, second edition. Cambridge University Press: Cambridge.

Crowe, C. C., Sanders, W. E., and Longley, S. (1973). Bacterial interference. II. Role of the normal throat flora in prevention of colonization by Group A Streptoccocus. Journal of Infectious Diseases, 128, 527-532.
deLouvois, J., Hurley, R., and Stanley, V. C. (1975). Microbial flora of the lower genital tract during pregnancy: relationship to morbidity. Journal of Clinical Pathology, 28, 731-735.

Galask, R. P., Larsen, B., and Ohm, M. (1976). Vaginal flora and its role in disease. Clinical Obstetrics and Gynecology, 19, 61-81.

Gordon, A. M., Hughes, H. E., and Barr, G. T. D. (1966). Bacterial flora in abnormalities of the female genital tract. Journal of Clinical Pathology, 19, 429-432.

Hipp, S. S., Lawton, W. D., Chen, N. D., and Gaafar, H. A. (1974). Inhibition of Neisseria gonorrhoeae by a factor produced by Candida albicans. Applied Microbiology, 27, 192-196.

Hite, K. E., Hesseltine, H. C., and Goldstein, L. (1947). A study of the bacterial flora of normal and pathogenic vagina and uterus. American Journal of Obstretics and Gynecology, 53, 233-240.

Holmberg, K., and Halkinder, H. O. (1972). Interference between gram positive organisms in dental plaque. Journal of Dental Research, 51, 588-595.

Hurley, R., Stanley, V. C., Leask, B. G. S., and deLouvois, J. (1974). Microflora of the vagina during pregnancy. In The Normal Microflora of Man, pp. 155-185. Edited by F. A. Skinner and J. G. Carr. Academic Press: London.

Kraus, S. J., and Ellison, N. (1974). Resistance to gonorrhoea possibly mediated by bacterial interference. Applied Microbiology, 27, 1014-1016.

Kraus, S. J., Geller, R. C., Perkins, G. H., and Rhoden, D. L. (1976). Interference of Neisseria gonorrhoeae growth by other bacterial species. Journal of Clinical Microbiology, 4, 288-295.

Levison, M. E., Corman, L. C., Carrington, E. R., and Kay, D. (1977). Quantitative microflora of the vagina. American Journal of Obstetrics and Gynecology, 127, 80-85.

Selwyn, S. (1975). Natural antibiosis among skin bacteria as a primary defence against infection. British Journal of Dermatology, $93,487-493$.

Sprunt, K., Leidy, G. A., and Redman, W. (1971). Prevention of bacterial overgrowth. Journal of Infectious Diseases, 123, 1-10. 